\title{
Electron Correlation in the Continuum
}

CONF-890708--4

DE89 015359

C. Bottcher and M. R. Strayer

Physics Division, Oak Ridge National Laboratory * Oak Ridge, TN 37831-6373 USA

Invited talk to be presented as part of a Symposium on Supercomputational Collision Physics

at the XVI International Conference on the Physics of Electronic Corlisions

New York, July 26 - August 1, 1989

July 24, 1989

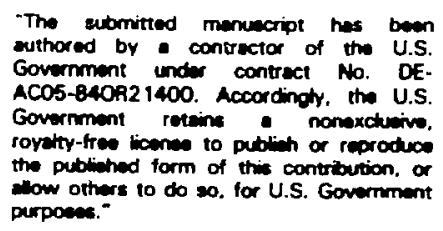

* Work performed under the auspices of the U.S. Department of Energy, Office of Basic Energy Sciences, Division of Chemical Sciences. Oak Ridge National Laboratory is operated by Martin Marietta Energy Systems under Contract No. DE-AC05-84OR21400 with the U.S. Department of Energy. 


\title{
Electron Correlation in the Continuum
}

\author{
Christopher Bottcher and Michael R. Strayer
}

Oak Kidge National Laboratory, Oak Ridge, Tennessee 37831, USA

\begin{abstract}
We consider a class of problems, notably double ionization, which require accurate descriptions of correlation in both the initia! and final states. Methods are presented for representing correlated wavefunctions on a basis spline lattice, and for calculating bound-continuum transition probabilities.
\end{abstract}

\section{Introduction: problems and approaches}

Double photoionization

$$
h \nu+\mathrm{He} \longrightarrow e+e+\mathrm{He}^{++}
$$

has long been recognized as the prototype of correlated atomic processes, in that it is obviously forbidden in a truly independent particle description. Thus it has become the test bench for theoretical methods which attempt to go beyond independent particle approximations. Interest has intensified with the advent of experiments which replace the photon by a fast projectile, in particular a highly charged ion. We shall give a preliminary report on a new approach to these problems, which we expect to improve on previous theories in two respects. First, the initial and final states are calculated in a consistent framework, and secondly, the angular and energy distributions of the outgoing electrons are extracted.

Let us briefly review the difficulties in the way of calculating the cross section of a process such as (1). If the photon or projectile is described by perturbation theory, we have to evaluate a matrix element

$$
\boldsymbol{M}_{f i}=<\Psi_{f}|D| \Phi_{i}>
$$

where $\Phi_{i}$ is the initial (bound) state, $\Psi_{f}$ the final (continuum) state and $D$ is a dipole matrix element, say

$$
D=z_{1}+z_{2} .
$$

More generally, $D$ would be a sum of rather complicated multipoles, but the principle is unaltered. At this level of approximation, only the final state wavefunction $\Psi_{f}$ poses any difficulties. If the excitation mechanism is a slow collision, final state and post-collision effects enter to make the calculation formidably more difficult.

The leading difficulty in constructing $\Psi_{f}$ for particles with Coulomb interactions at large distances has long been that of specifying the asymptotic boundary conditions. Much progress has recently been made on the boundary conditions ${ }^{1,2}$, but other problems remain. Most variational or $\mathrm{R}$ matrix prescriptions for calculating the short-range part of a scattering wavefunction with two unbound particles suffer from divergences, traceable to the free-particle Green function. Thus a complete calculation of $\Psi_{f}$ is some distance in the future. However the matrix element $M_{f i}$ should be calculable without a complete knowledge of $\Psi_{f}$, particularly in the asymptotic region. Successful descriptions of bound-continuum processes have been carried out by many authors using bound state techniques to describe the short-range part of $\Psi_{f}$. The idea in a rigorous form dates from Ref. 3 , and it has of course been applied in numerous papers since then 4

\section{Schrödinger equation for the three-body problem}

Since we wish to consider methods which have the possibility of converging to an accurate solution, we shall represent the dependence on the internuclear distance explicitly, rather than through configuration interaction. In this section we present the analysis needed for an accurate numerical representation of a two-electron wavefunction of given orbital angular momentum, spin and parity.

Consider two electrons moving in the field of an infinitely heavy nucleus of charge $C$. If the electrons have position vectors $\overrightarrow{r_{1}}, \overrightarrow{r_{2}}$, the (non relativistic) 
Hamiltonian is given by

$$
H=-\frac{1}{2}\left(\nabla_{1}^{2}+\nabla_{2}^{2}\right)+\left(\frac{1}{r_{12}}-\frac{C}{r_{1}}-\frac{C}{r_{2}}\right),
$$

where $r_{12}=\left(r_{1}^{2}+r_{2}^{2}-2 r_{1} r_{2} \cos \vartheta\right)^{\frac{1}{2}}$ and $\vartheta$ is the angle between $\overrightarrow{r_{1}}$ and $\overrightarrow{r_{2}}$.

The exact nonrelativistic wavefunction for a state of total spin $S$ and total orbital angular momentum $L$ can be expanded as follows,

$$
\begin{aligned}
\Psi\left(\overrightarrow{r_{1}}, \overrightarrow{r_{2}}\right) & =\Theta_{S M_{s}} \sum_{\ell=\varpi}^{L} \psi_{\ell}\left(r_{1}, r_{2}, \vartheta\right) \\
& \times \mathcal{Y}_{L M_{L}}(\ell, L+\varpi-\ell)
\end{aligned}
$$

where $\Theta$ is a spin eigenfunction, and $\mathcal{Y}_{L M_{L}}\left(\ell, \ell^{\prime}\right)$ are the usual coupled spherical harmonics. The switch $\varpi=0,1$ dictates the overall parity: $\Pi=(-1)^{L+\varpi}$. Then it is straightforward to derive a set of coupled equations satisfied by the functions $\psi_{\ell}$, whose arguments are the three "dynamical" variables $r_{1}, r_{2}$, and $\vartheta$,

$$
\begin{gathered}
\text { [h }(\ell, L+\varpi-\ell)-E] \psi_{\ell}= \\
\sum_{\ell^{\prime}}\left[v_{\ell \ell^{\prime}}^{(1)}+v_{\ell \ell^{\prime}}^{(2)}\right] \psi_{\ell^{\prime}}
\end{gathered}
$$

The diagonal Hamiltonian has the obvious form

$$
\begin{aligned}
\mathrm{h}\left(\ell_{1}, \ell_{2}\right) & =T\left[r_{1}\right]+T\left[r_{2}\right]+T[\vartheta] \\
& +\left[\frac{\ell_{1}\left(\ell_{1}+1\right)}{2 r_{1}^{2}}+\frac{\ell_{2}\left(\ell_{2}+1\right)}{2 r_{2}^{2}}\right],
\end{aligned}
$$

where $-2 T[r]=\partial^{2} / \partial r^{2}$ and

$$
-2 T[\vartheta]=\left(\frac{1}{r_{1}^{2}}+\frac{1}{r_{2}^{2}}\right) \frac{1}{\sin \vartheta} \frac{\partial}{\partial \vartheta}\left(\sin \vartheta \frac{\partial}{\partial \vartheta}\right) .
$$

The coupling terms are given by

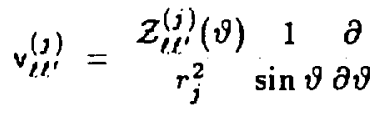

for $j=1,2$. General formulae for the coefficients $\mathcal{Z}$ as polynomials in $\cos \vartheta$ have been obtained from angular momentum theory. In addition to these equations, the functions $\psi_{\ell}$ possess symmetries under interchange of $r_{1}$ and $r_{2}$, depending on $S, L$ and $\varpi$. The analysis can be applied to three particles of arbitrary mass, if the coordinates are appropriately redefined.

\section{Discretization on a lattice}

We choose to represent the equations (6) on a discrele lattice of points in $r_{1}, r_{2}, \vartheta$ space by means of the Basis Spline Collocation technique. This method has been applied with success to the Schrodinger and Dirac equations in three cartesian coordinates in the context of ion-atom collisions, so its extension to a similar equation in another three-dimensional space is a natural one. The earlier Finite Element method was extensively applied to two electron systems, using the time dependent wavepacket approach ${ }^{5}$. Since the theory of the BSC method has been described in several papers ${ }^{6}$, we only state the underlying postulates and final working equations here, for completeness.

In a collocation method, an operator equation $L[\psi]=0$ is discretized by expanding $\psi(\vec{x})$ in a finite basis $u_{k}(\vec{x}), k=1, \ldots, K$, where the coefficients are determined by setting $L\left[\sum \psi^{k} u_{k}\right]=0$ at $N$ collocation points $\vec{\xi}_{\alpha}, \alpha=1, \ldots, N$. In practice each function is localized around a small subset of these points, and the coefficients $\psi_{k}$ are eliminated in favour of the values of the solution $\psi_{\alpha}$ at the points $\vec{\xi}_{a x}$. The resulting equations have the structure of finite difference algorithms, while drawing upon the full power of modern functional methods. We choose localized piecewise continuous polynomials, known as basis splines, as overall the most powerful and flexible interpolating functions yet devised.

The solutions of (6) are thus expanded in products of splines,

$$
\psi_{\ell}\left(r_{1}, r_{2}, \vartheta\right)=\sum_{i j k} c_{i j k} u_{i}\left(r_{1}\right) u_{j}\left(r_{2}\right) w_{k}(\vartheta),
$$

and the collocation principle is applied as described to obtain the equations satisfied by the vector of $\psi_{\ell}\left(r_{1<x}, r_{2 \beta}, \vartheta_{\gamma}\right)$ on the collocation lattice. Operators become matrices in this space. The potential energy, and indeed any local function of $r_{1}, r_{2}, \vartheta$, is diagonal in the indices $\alpha, \beta, \gamma$. Each term in the kinetic energy is a sparse block matrix, e.g. $T\left[r_{1}\right]$ has the structure $t_{\alpha, \gamma^{\prime}} \delta_{\beta, \beta^{\prime}} \delta_{\gamma, \gamma^{\prime}}$. This extreme sparseness gives the collocation method an advantage, particularly in three dimensions, over other formulations, such as that of Galerkin. We want the Hamiltonian to be sparse, since we do not wish to store its 
full matrix representation. Rather, all algorithms are broken down into canonical operations of the form: matrix $\times$ vector $\longrightarrow$ vector, where the matrix need only be represented implicitly by its non zero blocks.

Before proceding to the subject of linear operator methods, some technical aspects of the collocation methods are worthy of comment. As implied by (10), different bases are used for the radial and angular coordinates. To obtain accurate wavefunctions, these bases must satisfy precise boundary conditions which accommodate the cusp conditions at the nucleus, and when the two electrons are close together. The radial functions $u_{i}(r) \sim r-C r^{2}$, in the metric of (7). Thus we arrange that $u_{i}$ is a polynomial in $r,=0$ at the origin. Near $\vartheta=0$, the expansion $\psi \sim a+b \vartheta+\cdots$ fits the two-body cusp: $\psi \sim c\left(1+\frac{1}{2} r_{12}\right)$, while near $\vartheta=\pi$, the correct expansion is $\psi \sim a^{\prime}+b^{\prime}(\pi-\vartheta)^{2}$. These conditions are satisfied if $w_{k}(\vartheta)$ is a polynomial in the variable $\vartheta$ as opposed to $\cos \vartheta$, and $\neq 0$ at $\vartheta=0, \pi$.

Finally, we note that the collocation representation of the Hamiltonian is usually not self-adjoint, i.e. the kinetic energy matrices are not symmetric. They do satisfy the more general criterion of factorizing into a product $\mathbf{T}=\mathbf{S}^{-1} \tilde{\mathbf{T}}$ of Hermitian matrices, $\mathbf{S}$ being positive definite, a sufficient condition to ensure that the spectrum of $\mathbf{T}$ is real. A practical consequence is that normal and adjoint solutions must always be carried along together, e.g.

$$
(\mathbf{H}-E) \Psi=0,\left(\mathbf{H}^{T}-E\right) \tilde{\Psi}=0,
$$

where norms and inner products always have the form $\tilde{\mathbf{\Psi}}^{T} \mathbf{\Psi}$. Intuitively one may think of the adjoint vector as carrying a quadrature weight.

\section{Linear operator methods}

To obtain the initial and final states in (2), we use linear algebraic techniques taken over from the ion-atom problem with little modification ${ }^{6,7}$. We shall use the customary notation of quantum mechanics, provided it is understood that operators are malrices, kets are vectors and bras are vectors in adjoint space. Bound state solutions are calculated by damped relaxation methods. The solution of $\left(H-E_{\mathrm{n}}\right) \Phi_{n}=0$ is calculated by iterating

$$
\Phi^{(I+1)}=\Phi^{(I)}+\mathcal{D}\left(H-E^{(I)}\right) \Phi^{(I)}
$$

where $E=\langle H\rangle$ and $\mathcal{D}=\lambda(1+T / \mu)^{-1}$. In practice we construct an approximate $\mathcal{D}$ separable in the spaces of $r_{1}, r_{2}, \vartheta$. The constants $\lambda, \mu$ can always be chosen to guarantee rapid convergence. If excited states are required, one enforces orthogonality to all lower-lying states by projecting at each iteration. Indeed any desired constraint can be incorporated in this method, a notable example being Feshbach projection to calculate autoionizing states. Suppose $\omega_{q}$ are the bound states of the one body Hamiltonian embedded in (7), e.g. that of $\mathrm{He}^{+}$. The Feshbach projection operators are

$$
P_{q}=\left|\omega_{q}(1)><\omega_{q}(1)\right|+(1 \leftrightarrow 2) .
$$

If we project out the is state, by enforcing $P_{1}, \Phi=$ 0 , we can use (12) to calculate autoionizing states, e.g. $H e\left(2 s^{2}\right)$.

Turning now to the calculation of (2), we shall reformulate the problem slightly. Let $M_{f i}=M_{\lambda}(E)$ where $f$ is a continuum state of energy $E$, distinguished by other quantum numbers $\lambda$. Then

$$
D \Phi_{i}=\sum_{\lambda} \int d E M_{\lambda}(E) \Psi_{\lambda}(E) .
$$

Bound state contributions to (14) are omitted for clarity; we note that they are easy to calculate explicitly and subtract out. Now we introduce the Gaussian filter operator,

$$
F(E-H, \Delta)=(\sqrt{\pi} \Delta)^{-1 / 2} \exp \left[-\frac{(E-H)^{2}}{2 \Delta^{2}}\right],
$$

so normalized that $\int F^{2} d E=1$. Applying this operator to (15), elementary quantum mechanics shows that

$$
\begin{aligned}
<\chi(E, \Delta) \mid \chi(E, \Delta)> & =\sum_{\lambda} \int d \epsilon F(E-\epsilon, \Delta)^{2} \\
& \times\left|M_{\lambda}(\epsilon)\right|^{2}, \quad \text { (16) }
\end{aligned}
$$

where

$$
\chi(E, \Delta)=F(E-H, \Delta) D \Phi_{i} .
$$

For reasonably small values of $\Delta$, we can write simply

$$
<\left.\chi(E, \Delta)\left|\chi(E, \Delta)>\simeq \sum_{\lambda}\right| M_{\lambda}(E)\right|^{2} .
$$


The filter considered here is a functional of the full Hamiltonian: any other operator could replace $H$, leading to projections on a different spectrum. If the individual $\lambda$ terms could not be disentangled, this would not be a very interesting result: the sum is usually dominated by single ionization leaving the ion in its ground state. Fortunately we can do better, but it is necessary to look more closely at the properties of $\chi$.

For an $E>0,(7)$ defines a normalizable wavepacket of spatial extent $a \sim E^{1 / 2} / \Delta$. Thus for small $\Delta$ (such that $a<r_{q}$, the size of a bound state), $\chi$ can be represented over most of space by its asymptotic form

$$
\begin{aligned}
\chi & \sim \sum \omega_{q}(1) f_{q}^{(-)}(2) \\
& +\sum \omega_{q}(2) f_{q}^{(-)}(1)+\chi_{C}
\end{aligned}
$$

where $\omega_{q}$ are bound states of the ion as before, and $\chi_{C}$ is a superposition of true doubly ionized states. Thus if $\chi_{q}=P_{q} \chi$, in the notation of (13),

$$
<\left.\chi_{q}\left|\chi_{q}>=\right| M_{q}^{(1)}\right|^{2}
$$

the contribution from single ionization, leaving the ion in state $q$. The term $\chi_{c}$ in (19) is obviously given by $\prod\left(1-P_{q}\right) \chi$, whence

$$
\left\langle\chi_{C} \mid \chi_{C}\right\rangle=\sum_{\lambda}\left|M_{\lambda}^{(2)}\right|^{2},
$$

the true double ionization contribution.

We emphasize that these calculations are valid for a lattice Iarger that a bound atom, though finite. We also recall that the spline basis can represent continuum states on such a lattice very well. Our procedure should be contrasted with the incorrect one of projecting (14) before filtering, i.e. calculating $F P_{q} D \Phi_{i}$ instead of $P_{q} F D \Phi_{i}$. Since $P_{q}$ is a functional of the independent electron part of the Hamiltonian, it does not commute with $F$. This is an aspect of the well recognized fact ${ }^{8}$ that projection from a configuration interaction representation by itself cannot distinguish single from double ionization. In summary, projection as in (20) is valid because the Gaussian filter picks out the asymptotic region. In particular, (20) does not contain contributions from autoionizing states. The filter plays a role in lattice calculations analogous to that of the Green function in Hilbert space.

We have finally to deconvolute different contributions to the double ionization contained in the wavepacket $\left|\chi_{C}\right|^{2}$. The analysis of a wavepacket of two unbound electrons was discussed in Ref. 5 . Let us express $\left|\chi_{C}\right|^{2}$ in the hyperspherical coordinate system $R, \alpha, \vartheta$, where $r_{1}=R \cos \alpha_{1} r_{2} \sin \alpha$. Consider the probability in a cone defined by $\alpha_{0}-$ $\Delta \alpha / 2<\alpha<\alpha_{0}+\Delta \alpha / 2, \vartheta_{0}-\Delta \vartheta / 2<\vartheta<$ $\vartheta_{y}+\Delta \vartheta / 2$, and $0<R<R_{\max }$. If the electrons are only weakly interacting in most of the space, this probability (divided by $\Delta \alpha \Delta \vartheta$ ) is the amplitude associated with the two-electron state $\alpha_{0}, \vartheta_{1,}$; in other words, the electrons are ejected at a relative angle $\vartheta$ and with energies $E_{1}, E_{2}$ such that $E_{1} / E_{2}=\tan ^{2} \alpha_{U}$. The proof of this result from scattering theory is rather lengthy, and our remarks here are only intended as a heuristic justification.

\section{Numerical calculations}

The numerical implementation of (17) was described in detail in Ref. 9 . There we evaluated the Gaussian by a splitting technique: letting $Z=$ $(E-H)^{2} / \Delta^{2}$ we write $\exp (-Z)=[\exp (-Z / M)]^{M}$, where $M$ is sufficiently large that $\exp (-Z / M)$ can be expanded in a Taylor series. Though effective, this is a very slow procedure, so we have replaced the Gaussian by a rational function

$$
F=\mathcal{N}\left(1+\frac{Z}{M}\right)^{-M}
$$

where $\mathcal{N}$ is the normalizing factor. The operator in (22) is inverted by a damped relaxation method, similar to (12): to solve $(1+Z / M) \xi=\eta$ we iterate

$$
\begin{aligned}
\xi^{(I+1)} & =\xi^{(I)}+\mathcal{D}^{2} \rho^{(I)} \\
\rho^{(I)} & =\eta-(1+Z / M) \xi^{(I)} .
\end{aligned}
$$

Notice that $\mathcal{D}$ is squared to cancel $H^{2}$ in $Z$. Our revised procedure is hundreds of times faster than the old.

The methods outlined in Section IV are novel, but fortunately they can be tested thoroughly. The use of the filter to extract matrix elements (16) has been applied to a variety of soluble single channel problems ${ }^{9}$. We have carried out similar tests for 
the multichannel cases of interest here. A crucial issue is the validity of the projection technique underlying (19)-(21). If the postulate (19) is valid the calculated amplitudes shouid be independent of $\Delta$, which they are to a good approximation. We can also check sum rules, such as

$$
\int d E \sum_{\lambda}\left|M_{\lambda}(E)\right|^{2}=<\Phi_{1}\left|D^{2}\right| \Phi_{1}>.
$$

In general our calculated total amplitudes for double ionization and single ionization with simultaneous excitation are in accord with earlier accurate calculations ${ }^{10,11}$, and results will be presented elsewhere. The differential amplitudes for double ejection show strong correlations between the two electrons as predicted by the Wannier theory ${ }^{1,5}$, and these correlations persist to energies well above threshold.

\section{Conclusions and prospects}

We have presented a unified description of scattering processes involving electronic correlation in both bound and continuum states. Many other processes can be treated by the same methods. A simple example is provided by double excitation, usually leading to an autoionizing state

$$
\mathcal{Z}+\mathrm{He} \longrightarrow \mathcal{Z}^{\star}+\mathrm{He}\left(q q^{\prime}\right)
$$

where $\mathcal{Z}$ stands for any fast projectile, and the final state of Helium is labelled by two single electron orbital designations $q, q^{\prime}$ (e.g. $2 s, 2 p$ ). The quantum numbers of the final state in (24) including its allignment can be determined experimentally. Theoretical interest focusses on the competition between single and multiple step mechanisms ${ }^{12}$.

Another class of problems involves the decay of autoionizing states in external fields. As before, we require bound-continuum matrix elements,

$$
\mathcal{V}_{\lambda}(E)=<\Psi_{\lambda}(E)|H-E+D| \Phi_{g g^{\prime}}>
$$

where $D$ is the operator of the external field. The autoionizing state $\Phi_{g q^{\prime}}$ is calculated by projection, and the continuum state $\Psi_{\lambda}(E)$ describes the outgoing electrons. The angular distribution of these electrons is of great experimental interest ${ }^{13}$, but has been difficult to predict because of its extreme sensitivity to correlation in the wavefunctions.
Time dependent processes can also be addressed, insofar as they can properly be desr-ibed by a limited number of angular momentum eigenstates. A problem of pedagogical interest is to monitor the decay of autoionizing states in time, a process which remains intuitively mysterious, in spite of its long history.

\section{Acknowledgements}

This research was supported by the U.S. Department of Energy, Office of Basic Energy Sciences, Division of Chemical Sciences. Oak Ridge National Laboratory is operated by Martin Marietta Energy Systems under Contract No. DE-AC05-84OR21400 with the U.S. Department of Energy.

\section{References.}

1. C. Bottcher, Adv. Atom. Molec. Phys. 25, 303 (1989).

2. P. L. Altick, Phys. Rev. A25, 128 (1982).

3. Y. M. Chan and A. Dalgarno, Proc. Phys. London 85, 277 (1965).

4. H. S. Taylor and A. U. Hazi, Phys. Rev. A14, 2071 (1976).

5. C. Bottcher, Adv. Atom. Molec. Phys, 20, 241 (1985).

6. C. Bottcher and M. R. Strayer, Ann. Phys. (N.Y.) 175, 64 (1987).

7. C. Bottcher, M. R. Strayer, A. S. Umar and P. G. Reinhardt, Phys. Rev. A, in press (1989).

8. A. L. Ford and J. F. Reading, J. Phys. B21, L685 (1988).

9. C. Bottcher, M. R. Strayer, A. S. Umar and V. E. Oberacker, Phys. Rev. C37, 2487 (1988).

10. S. L. Carter and H. P. Kelly, Phys. Rev. A24, $170(1981)$.

11. M. R. H. Rudge, J. Phys. B21, 1887 (1988).

12. J. P. Giese, M. Schultz, J. K. Swenson, H. Schöne, S. L. Varghese, C. R. Vare, M. Benhenni, P. F. Dittner and S. Datz, contributed paper no. 484, this conference (1989).

13. J. K. Swenson, C. C. Havener, N. Stolterfoht, K. Sommer and F. W. Meyer, Phys. Rcy. Lett. 63, 35 (1989). 


\section{DISCLAIMER}

This report was prepared as an account of work sponsored by an agency of the United States Government. Neither the United States Government nor any agency thereof, nor any of their employees, makes any warranty, express or implied, or assumes any legal liability or responsibility for the accuracy, completeness, or usefulness of any information, apparatus, product, or process disclosed, or represents that its use would not infringe privately owned rights. Reference herein to any specific commercial product, process, or service by trade name, trademark, manufacturer, or otherwise does not necessarily constitute or imply its endorsement, recommendation, or favoring by the United States Government or any agency thereof. The views and opinions of authors expressed herein do not necessarily state or reflect those of the United States Government or any agency thereof. 